\title{
La gestión y la promoción cultural en los municipios distritales de Lima
}

\author{
Cultural management and promotion in the district \\ municipalities of Lima
}

\begin{abstract}
RESUMEN
El objetivo de este artículo es analizar y reflexionar en qué medida la gestión municipal afecta a la promoción cultural de centros culturales municipales de los distritos de Miraflores, San Isidro y Ate en el año 2017. Para ello, se analiza la organización interna, el capital humano y la planificación que afecta a la gestión municipal en la promoción cultural de los centros culturales municipales. En este artículo se buscará analizar tres gestiones culturales de tres distritos de Lima Metropolitana utilizando el benchmarking para encontrar fortalezas y cómo estas ayudarían en el fortalecimiento de cada uno, para recabar la información se utiliza entrevistas a los puestos gerenciales, las memorias, y documentos oficiales de cada municipalidad. Finalmente, el artículo presenta los resultados que se logró recabar de cada municipio en tres campos que serían la organización interna, la planificación y el potencial humano. Además, se comparan dichos resultados, dando reflexiones y conclusiones respectivas de cada campo.
\end{abstract}

Palabras clave: Promoción cultural; gestión cultural; consumo cultural; medios de comunicación; marketing cultural.

\begin{abstract}
The objective of this article is to analyze and reflect to what extent municipal management affects the cultural promotion of municipal cultural centers in the districts of Miraflores, San Isidro and Ate in 2017. For this, the internal organization, the capital, is analyzed. human and planning that affects municipal management in the cultural promotion of municipal cultural centers in the districts of Ate, Miraflores and San isidro. This article will seek to analyze three cultural activities in three districts of Metropolitan Lima using benchmarking to find strengths and how these would help to strengthen each one. To collect information, interviews with management positions, reports, and official documents are used. of each municipality. Finally, the article presents the results that were obtained from each municipality in three fields that would be internal organization, planning and human potential. Furthermore, these results are compared, giving reflections and respective conclusions of each field.
\end{abstract}

Keywords: Cultural promotion; cultural management; cultural consumption; media; cultural marketing.

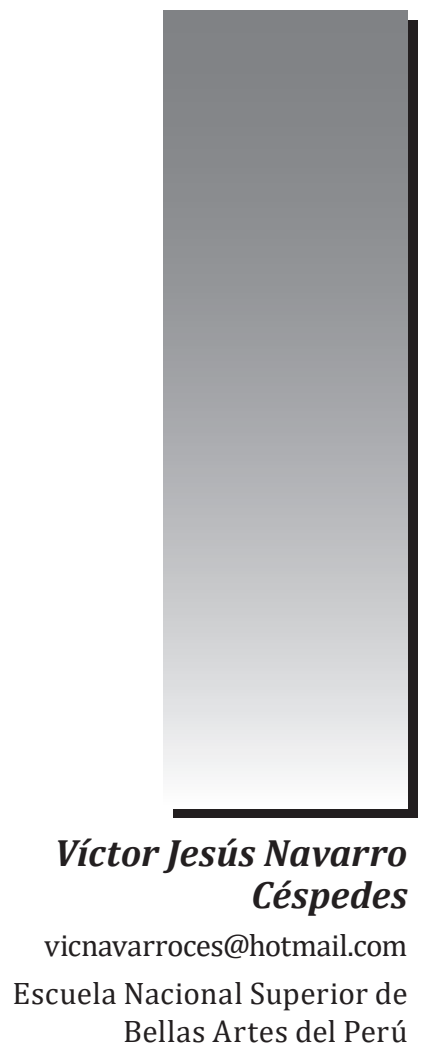

Presentado: 13/03/2020 - Aceptado: 29/10/2020 - Publicado: 26/11/2020

(C) Los autores. Este artículo es publicado por Gestión en el Tercer Milenio de la Facultad de Ciencias Administrativas de la Universidad Nacional Mayor de San Marcos. Este es un artículo de acceso abierto, distribuido bajo los términos de la licencia Creative Commons Atribucion - No Comercia_Compartir Igual 4.0 Internacional. (http://creativecommons.org/licenses/by-nc-sa/4.0/) que permite el uso no comercial, distribución y reproducción en cualquier medio, siempre que la obra original sea debidamente citada. 


\section{INTRODUCCIÓN}

En el año 2016, los habitantes de Lima Metropolitana que pertenecen a los sectores socioeconómicos A y B realizan mayor cantidad de actividades recreativas y culturales que aquellos que son parte de los sectores socioeconómicos D y E (Lima Cómo Vamos, 2017). Entre las muchas actividades que se señalan en la encuesta se puede señalar un ejemplo para mostrar dicha diferencia, los sectores socioeconómicos $\mathrm{A}$ y $\mathrm{B}$ son los que más visitan museos o galerías (31.5\%), a diferencia de los sectores socioeconómicos D y E (6,9\%) (Lima Cómo vamos, 2017). De esta manera se presenta la misma tendencia en las otras actividades como visitar monumentos o lugares históricos o arqueológicos, asistir al teatro, asistir a conferencias o seminarios, entre otros. A partir de este dato estadístico, se pueden observar una serie de problemas relacionados tanto a la demanda como a la oferta de estas actividades recreativas y culturales. En este artículo, vamos a abordar aquellos problemas relacionados con la oferta cultural y recreativa, centrándonos en los municipios que trabajan, considerando el marco nacional dado por el Ministerio de Cultura, en mejorar las oportunidades de recreación y cultura.

Por esta razón, se consideraron tres municipios: Ate, Miraflores y San Isidro. Estos tres distritos poseen centros culturales que desarrollan actividades de promoción cultural. Sin embargo, poseen diversas formas de aproximarse al tema cultural y recreativo, asimismo poseen distintas realidades. Según el Instituto Nacional de Estadística e Informática (INEI, 2016), el distrito de Ate posee un porcentaje de $11.5 \%$ personas cuyo estrato es alto (ingresos per cápita por hogares 2192.20 a más nuevos soles) y medio alto (ingresos per cápita por hogares 1330.10 a 2192.19 nuevos soles); por otro lado, un $62.8 \%$ posee un estrato medio bajo (ingresos per cápita por hogares 575.70 a 898.99 nuevos soles) y bajo (ingresos per cápita por hogares menor de 575.69 nuevos soles). Mientras que tanto el distrito de Miraflores como San Isidro poseen una mayoría de personas que están en el estrato alto (ingresos per cápita por hogares 2192.20 a más nuevos soles), $94.4 \%$ y $100 \%$. Por estas razones, lo importante en este estudio es señalar dichas características que los diferencia y compararlas con el fin de señalar deficiencias y aciertos.
Cabría señalar tres conceptos que se conectan entre sí, tales como la política cultural, la gestión cultural y la promoción cultural. La cultura se puede dividir en dos grandes definiciones, que la política cultural debe responder; la primera, es aquella que hace referencia al modo de vida de una comunidad (creencias, cosmovisiones, costumbres, símbolos y prácticas); y la segunda, hace referencia a un conjunto de objetos y prácticas, a obras de arte o expresiones artísticas (Ministerio de Cultura, 2012). Por ello, señala el Ministerio de Cultura (2012) que la política cultural "es un conjunto de orientaciones, normativas y proyectos que están destinados a democratizar la producción, la circulación y el consumo de objetos y servicios culturales" (p. 7). De esta manera, la política cultural debe estar enfocada en que esta oferta cultural, sea tanto generada como consumida por cualquier habitante sin distinción de ningún nivel, lo que generará en la población una identidad cultural cohesionada.

Esta política cultural debe plasmarse en la realidad, esto conlleva una serie de acciones, que comprende la gestión cultural. Se entiende por gestión cultural:

el conjunto de acciones de dirección, coordinación, planificación, evaluación, seguimiento y ejecución destinadas a facilitar, promover, estimular, conservar $\mathrm{y}$ difundir las diferentes actividades $\mathrm{y}$ manifestaciones culturales en condiciones de libertad y equidad, orientadas a fomentar el ejercicio de derechos, el acceso a oportunidades y el mejoramiento de los estados de bienestar de las personas. (Ministerio de Cultura de Colombia, 2013, p. 10)

Entre las actividades que una gestión realiza, encontramos aquellas que buscan relacionar a la población con la cultura, el cual es un proceso comunicativo y de participación, definido como promoción cultural (Deriche, 2006). Este concepto tiene una relación directa con otros dos; el producto cultural y las audiencias culturales. El primero, señala que "no ocupa un lugar físico, sino ideal, donde el espectador se apropia cognitivamente de él, por encima de los significados o ideologías que se le asocien con vistas, por ejemplo, a unas ventas masivas" 
(Luengo, 2008, p. 327). Por otro lado, las audiencias culturales son definidos como:

Los destinatarios de la producción cultural (sin su presencia y apoyo la difusión perdería su sentido), y en un momento de crisis son las más fieles a la hora de contribuir con sus recursos a la financiación de la oferta. Además, su presencia legitima el resto de los recursos públicos y privados que acaban haciendo viable la programación cultural. (Antoine, 2011, párr. 1)

Para que la promoción cultural sea efectiva, es necesario que se aplican técnicas de marketing cultural, que en el sector público tiene en cuenta a los directivos que se enfrentan a un mercado mucho más complejo, dado que los ciudadanos individualmente juegan diversos roles en su relación con lo público (Losada, 1999).

Como señalamos líneas arriba, el estudio analiza tres gestiones municipales con el fin de observar cómo afecta la calidad de la promoción cultural en cada uno de ellos durante el año 2017. Para ello, específicamente se analizan cuatro áreas: la organización interna, el capital humano, la planificación y las actividades que afectarían la calidad de la promoción cultural en las gestiones de los tres distritos municipales, observando sus falencias y fortalezas y utilizando el método de benchmarking para señalar mejoras.

Este estudio aporta a una temática, la gestión cultural municipal en el Perú, donde hay poca bibliografía ${ }^{1}$. Finalmente, esta investigación beneficia no solo a las entidades municipales a las cuales analiza, sino también a los ciudadanos, puesto que se busca entender cómo se gestionan y promocionan los proyectos culturales que consumen.

\section{MÉTODOS}

Para este estudio se utilizó un método comparativo: el benchmarking ${ }^{2}$. Este método es un proceso de evaluación que analiza y compara con otras organizaciones o empresas líderes,

1 Hay que señalar que si existen muchos archivos bibliográficos que tocan indirectamente el tema, y que son considerados en esta investigación.

2 Según Arévalo y Cerro (2004), una posible traducción de este término al castellano sería "análisis técnicos competitivos". con el fin de que esta información pueda ayudar a la organización a mejorar su performance y rendimiento (Arévalo \& Cerro, 2004). Por ello, el proceso de Benchmarking conlleva planificación, recolección de datos, análisis estratégico y finalmente un proceso de adopción y adaptación que no se incorpora en este estudio, en el cual se comparan tres centros culturales de tres distritos de Lima Metropolitana. Así que se buscó analizar las fortalezas y falencias, con el fin de poder señalar aquello que podría fortalecer cada centro cultural para mejorar la difusión y promoción en el consumo de productos culturales durante el periodo 2017.

Para recabar la información se utilizaron técnicas de investigación cualitativas como lo son las tres entrevistas a los puestos gerenciales; la revisión de documentos oficiales como las memorias, Plan Estratégico Institucional (PEI), Plan Operativo Institucional (POI) y los Cuadros para Asignación de Personal (CAP) de cada municipalidad. Además, se recopiló el número de actividades, personal de área y presupuesto.

\section{RESULTADOS}

\section{Presupuesto.}

Las municipalidades de Ate, San Isidro y Miraflores cuentan con un presupuesto asignado para la promoción y difusión de sus actividades para el año 2017. Según el Ministerio de Economía y Finanzas (MEF, 2018), las municipalidades de Ate, Miraflores y San Isidro asignaron 3 706 248, 6468 416, y 6204860 nuevos soles, respectivamente. De esta manera, la mayor asignación del presupuesto la tiene la municipalidad de Miraflores, y la menor, la municipalidad de Ate.

\section{Organización.}

Las tres municipalidades cuentan con una gerencia, que entre sus funciones se encargan del tema de cultura en su respectivo distrito para el año 2017. La municipalidad de Ate cuenta con la gerencia de desarrollo social y cultural, subdividida en la subgerencia de desarrollo humano y cultural, la subgerencia de programas sociales e inclusión social, y la subgerencia de participación ciudadana. Además, cuenta con un comité distrital del patrimonio cultural de Ate, entidad consultiva cuya organización fue 
aprobada por Ordenanza $\mathrm{N}^{\circ}$ 409-MDA, y que consta de nueve miembros.

A diferencia de Ate, las municipalidades de Miraflores y San Isidro cuentan con una gerencia de Cultura y Turismo que se encargan de organizar y ejecutar las actividades de ambos rubros. Están a cargo de un gerente y no tienen sugerencias.

\section{Capital humano.}

Para poder señalar la estructura de las áreas o gerencias que se encargan del tema específico de cultura, observamos el Cuadro de Asignación de Personal (CAP) de cada municipalidad, y cotejamos la información con lo que nos señalaron los gerentes entrevistados.

La municipalidad de Ate según su CAP del año 2008, que es el único que se encuentra en su página web, señala que hay una gerencia de servicios sociales. Sin embargo, en conversaciones con la gerencia de desarrollo social y cultural, señalan que existe una asignación de cargos interna que no ha sido publicado ni se ha aprobado mediante ningún documento oficial, durante el año que se realizó la investigación, señalando que se cuenta con siete personas. Asimismo, cabe indicar, que las agencias cuentan cada una con dos trabajadores que se encargan de todas las áreas y no solamente de Cultura (Municipalidad de Ate, 2008). Además, el personal tiene capacitaciones en programas y temas de desarrollo social porque era prioridad del distrito. Por tal motivo para no dejar de lado el tema de cultura, señalan se capacitó al personal en temas de gestión cultural. Por esta razón, desde un inicio no todo el personal encargado del área cultural era profesional en el tema de cultura.

Según la municipalidad Metropolitana de Miraflores (2011), el distrito cuenta para la gerencia de cultura y turismo con un total de once personas. Además, no se han realizado capacitaciones internas debido a que solicitan dentro de los requisitos que el personal contratado tenga capacidades y habilidades del área. Desde un inicio en la gestión, la gerencia ya contaba con personal con estudios y experiencia en cultura.

La municipalidad de San Isidro ha aprobado un CAP el año 2019 (Ordenanza N 510-MSI), donde la gerencia de Cultura tiene consignados once trabajadores. Además, la gerencia realiza capacitaciones a su personal llevándolo a los talleres, seminarios o ponencias que ofrece el ministerio de cultura y la biblioteca nacional para poder actualizarse. La gerencia, al igual que en Miraflores, señala los requisitos de capacidades y habilidades dentro del área de cultura al contratar personal bajo la modalidad de Contrato Administrativo de Servicios (CAS), y por tal motivo buscan trabajadores que tengan dominio en el área de cultura.

\section{Planificación.}

Las municipalidades de Ate, Miraflores y San Isidro cuentan con lineamientos del distrito que guían sus planes operativos institucionales en el tema de Cultura.

La municipalidad de Ate, en el Plan Estratégico Institucional de Ate 2015-2018, señala en su línea estratégica $\mathrm{N}^{\circ} 2$ :

"Promover, difundir las manifestaciones artísticas y el legado histórico, los personajes de la cultura, patrimonio histórico y el desarrollo de actividades recreativas y turísticas" (Municipalidad de Ate, 2015, p. 42).

La municipalidad de Miraflores, en su Plan Estratégico Institucional 2011-2015, señala en su Objetivo Estratégico 3, estrategia 3,3:

"Fortalecer la identidad local, conversando, recuperando y difundiendo el patrimonio y tradiciones culturales en Miraflores, elaborando una programación diversa con gran valor artístico que fortalezca la presencia, tradición cultural y patrimonio del distrito" (Municipalidad de Miraflores, s/f., p. 111)

La municipalidad de San Isidro, en su Plan Estratégico Institucional 2015-2017, señala en su Objetivo 2:

"Incrementar la calidad y cantidad de los servicios culturales, sociales y deportivos y de salud".(Municipalidad de San Isidro, 2015, p. 8)

Además, las tres municipalidades realizan diagnóstico y planes; para ello señalan las siguientes actividades:

- La municipalidad de Ate realiza su diagnóstico utilizando técnicas como focus group, encuestas, boca a boca y un estudio de mercado. Además, realizan reuniones 
del equipo y deciden las actividades del centro cultural y de las agencias que tendrán dentro del año.

- La municipalidad de Miraflores cuenta con un plan estratégico que es elaborado por la gerencia encargada.

- La municipalidad de San Isidro, debido a que no cuentan con un plan estratégico normativo y público, ellos realizan un plan estratégico interno que busca lograr la optimización de sus objetivos, dentro de su plan, la gestión tiene metas de corto, mediano y largo plazo de manera cuantitativa.

\section{Actividades culturales.}

La municipalidad de Ate (2018) ha desarrollado actividades culturales dirigidas a niños y jóvenes como desfiles escolares, lecturas recreativas, beneficiarios de las bibliotecas municipales, talleres vacacionales, actividades culturales juveniles en los espacios públicos, y Festivales. Para adultos mayores: Danzas folklóricas y paseos turísticos.

Sin embargo, estas no son todas las actividades puesto que tiene un centro cultural y agencias municipales (Huaycán, Santa Clara y Salamanca). El primero realizó exposiciones, concursos culturales y presentaciones artísticas y culturales, que tuvo más de 11000 beneficiarios. Las segundas realizaron concursos, ferias de creatividad, ferias gastronómicas, y exposiciones.

La municipalidad de Miraflores (2018) ha desarrollado actividades culturales en sus salas de exposiciones (Miró Quesada y del Centro Cultural Ricardo Palma), Programas (relacionados con las artes visuales y escénicas, la formación de público, incentivos a la creación, familias en escena, y exprésate con Miraflores), y espacios culturales (Anfiteatro Chabuca Grande y otros espacios públicos descentralizados). Además, promociona los libros y la lectura con programas como Miraflores Lee, sus bibliotecas, programas para personas con discapacidad visual; y promociona el patrimonio cultural del distrito (museo de sitio Huaca Pucllana, Casa museo Ricardo Palma, y el museo Andrés Avelino Cáceres).
La municipalidad de San Isidro (2018) realiza diversas actividades en su centro cultural del Olivar (Teatro infantil y para adultos, conciertos musicales, talleres y exposiciones de arte), a través de su programa Cultura Libre (lleva cultura a las calles a través de una serie de actividades como cine, teatro y música), en sus espacios públicos con música (conciertos), en la biblioteca municipal y en la casa de la cultura (talleres y exposiciones).

\section{Promoción Cultural.}

La municipalidad de Ate tiene un plan de promoción que se basa en el boca a boca y las redes sociales, y no cuenta con una línea gráfica institucional. En relación con los medios audiovisuales, envía notas de prensa, y no tiene presupuestado hacer promoción impresa.

La municipalidad de Miraflores cuenta con un manual de procedimientos, en el cual se señalan como elaborar material gráfico y promocional (fotografías y medios audiovisuales), asesoría técnica especializada, la biblioteca, las donaciones, desarrollo y mantenimiento de colecciones y la evaluación de solicitudes para la utilización del auditorio del centro cultural. Además, utiliza medios impresos (solo en actividades expositivas, y para adultos mayores y zonas de menos recursos), envía notas de prensa a medios privados y estatales, cuenta con un canal llamado Mira Tv, y utiliza las redes sociales.

La municipalidad de San Isidro cuenta con una línea gráfica de la municipalidad, pero no una propia para el área cultural. Además, cuenta con una guía de actividades que es repartida a cada vecino, y un periódico municipal impreso; envía notas de prensa a medios privados y estatales y utiliza las redes sociales.

\section{DISCUSIÓN}

La promoción cultural se ve influenciada por la calidad de la gestión cultural de cada uno de los distritos, de esta manera, podremos observar como la promoción cultural del distrito de Miraflores es mejor, porque cuenta con una gestión cultural competente en los rubros que hemos analizados, asimismo, utilizando el Benchmarking podemos señalar como las otras gestiones culturales municipales pueden retroalimentarse de esta experiencia y estrategias. 
En relación con la organización, las municipalidades de Miraflores y San Isidro cuentan con una gerencia de cultura y turismo, lo que genera un mayor enfoque en desarrollar el área de cultura, ligándolo al turismo de la zona. Esta situación genera incentivos para los emprendimientos culturales. Por otro lado, la municipalidad de Ate presenta una serie de dificultades que son propios de este distrito y que cabría señalar como la extensión del distrito y su realidad socioeconómica (el $68.8 \%$ de su población posee ingresos per cápita menores a 898.99 soles), siendo que esta situación genera una mayor prioridad en programas de desarrollo social y que su centro cultural y las agencias tengan que buscar recursos de autogestión que es de montos variables y no confiables, debido a esto la calidad de la gestión de la promoción cultural disminuye considerablemente, así que para optimizar la organización y para mejorar la calidad de promoción cultural, la municipalidad de Ate podría formar la gerencia de cultura y turismo para enfocar su presupuesto y así ligándolo al turismo podrán generar un mayor incentivo para emprendimientos culturales y la promoción de lugares culturales dentro del distrito.

Aunque cabe señalar que la Municipalidad de Miraflores posee la mejor gestión cultural de las tres, y podemos observarlo en su oferta cultural, la cual es variada y atractiva, además sus actividades se desarrollan en espacios cerrados (espacio cultural, museo, biblioteca, entre otros) como espacios abiertos (espacios públicos).

Las tres gerencias concuerdan que les faltan trabajadores para lograr a cabalidad sus metas. En el caso de Ate, la situación es más complicada, puesto que por la extensión del distrito y al tener mayor población tienen agencias municipales, que cuentan con una o dos personas que se encargan de todas las funciones, incluido cultura, por tal motivo las tres municipalidades podrían incorporar especialistas en cultura, y la municipalidad de Ate podría incorporar las experiencias de San Isidro y Miraflores relacionados a capacitaciones sobre cultura.

La municipalidad de Miraflores cuenta con un manual de procedimientos y de marca de distrito, a diferencia de las otras dos municipalidades por lo cual esto permite que la gestión cultural tenga un orden y parámetros a pesar de esto, consideran que puede haber mejoras, en la medición profunda del impacto de las actividades dentro del distrito, con lo que podrían reformularlas, mejorarlas o mantenerlas, y con ello aumentar la calidad de la promoción cultural, con relación a las otras dos municipalidades, estas pueden elaborar e implementar manuales de procedimiento y de marca del distrito. Este tema es sumamente importante, pero está conectado a los otros dos (organización y capital humano), siendo que la municipalidad de San Isidro está más cercana, por otro lado, la municipalidad de Ate y Miraflores podría considerar utilizar las estrategias de promoción de la lectura que ha realizado San Isidro para reforzar su área de biblioteca.

La gestión cultural de los distritos de Miraflores, San Isidro y Ate impacta en la promoción cultural de cada una de las municipalidades, por tal motivo esto lo podemos observar en los instrumentos que cada una utiliza, y en el nivel de formalización, que depende del presupuesto, la organización, el capital humano y de la planificación, es por ello, que podemos señalar que Miraflores posee una mejor promoción cultural, puesto que utiliza como instrumentos una serie de medios como son las redes sociales, medios impresos y medios audiovisuales, que se sostienen en una sólida gestión cultural ${ }^{3}$. Así pues cuentan con una línea gráfica específicamente para el área de Cultura y que está en su misma planificación.

Por otro lado, San Isidro también cuenta con una buena gestión cultural, sin embargo, puede implementar algunas mejoras, relacionadas a la incorporación de una línea grafica para el área de cultura, y no depender de la que se realiza a nivel de toda la gestión municipal, también cuentan con una guía de actividades impresas que se reparte a cada vecino por lo cual tiene más llegada.

En Ate, con todos los problemas de gestión señalados, basa su estrategia de marketing cultural en redes sociales, de esta manera, se limita solo a una porción de los vecinos que cuentan con una red y dispositivos móviles, además, no cuenta con un seguimiento de sus actividades, solo se realiza una revisión superficial de la 3 La cual como señalan ellos mismos puede mejorar. 
cantidad de "like" por publicación y de la asistencia de la actividad, para finalizar, no cuentan con una sola línea gráfica si no tiene varias debido a que tiene diferentes organizadores en cada actividad y cada uno hace su propia línea gráfica.

\section{CONCLUSIONES}

El objetivo de esta investigación fue señalar la relación que existe entre la gestión municipal y la promoción cultural. A través de esta técnica comparativa de Benchmarking, el cual solo se llegó hasta la fase de análisis estratégico, considerando a la municipalidad de Miraflores como gestión a referenciar. Aunque en algunos puntos también se consideraron los avances de las otras gestiones para complementar.

A las municipalidades se les pidió que nos dieran información sobre la organización interna, el capital humano y la planificación que tienen sus gestiones municipales y culturales. Además, información sobre la promoción cultural.

Hemos señalado en la discusión que la gestión de cada una de las municipalidades afecta en la promoción cultural. De esta manera, la municipalidad de Miraflores cuenta con una sólida gestión cultural (una gerencia de Cultura y Turismo, personal capacitado en temas de cultura y un manual de procedimientos y de marca en el tema de cultura) y, por ende, su promoción cultural se expresa en diversos instrumentos y estrategias (medios impresos, audiovisuales y redes sociales), llegando no solo a los vecinos de su distrito sino también de otras partes de la ciudad.

De esta manera, las municipalidades de Ate y San Isidro, a través de un análisis comparativo, podrían incorporar, como lo señalamos en discusión, algunos de los lineamientos, acciones y estrategias que la municipalidad de Miraflores viene implementando. Desde la organización, un mayor presupuesto a este tema y una gerencia que se encargue directamente del tema, lo que supondría un cambio en la visión general sobre este tema en el distrito; el capital humano capacitado; y la planificación con metas a largo, mediano y corto plazo encadenados, asimismo, manuales que permitan establecer un orden en la forma como promocionan su oferta cultural, y como articulen con el sector privado.

Esta investigación todavía puede ser mejorada tanto en la ejecución o realizando varios benchmarking entre otras municipalidades. El tiempo de espera de la solución depende de las gestiones de las municipalidades y el grado de comunicación entre ellas. Concluimos que la metodología del benchmarking es un gran aporte para mejorar el nivel de desarrollo cultural y son más realistas para nuestra sociedad.

\section{Recomendaciones.}

Dentro de este artículo, siempre se desea que haya una mejora continua del mismo; por lo tanto se recomienda a futuros investigadores que tengan interés en el artículo, la complementación del sistema con más distribuciones para la demanda y el tiempo de espera, y aún más recomendable sería la implementación de este modelo en las distintas gestiones municipales que aportaran en el proceso de optimización, para hacer comparaciones entre los resultados arrojados por estas.

Se recomienda que se incluyan más municipalidades, para que el sistema se adapte y se replique a las necesidades de una mayor variedad de distritos. Para ello, se podría realizar una red conjunta entre municipios, es decir diálogos de comunicación entre ellas. Puesto que en este momento se encuentran aisladas, y esto produce que algunas municipalidades se estanquen o haya un desbalance en el desarrollo de la cultura.

Las tres municipalidades analizadas en este artículo deberían asignarle un mayor porcentaje de su presupuesto a la promoción cultural de su distrito, sobre todo la que ha designado menos recursos, porque tendrán más recursos para sus programas y evaluación, por lo cual esto generaría una mejor y más especializada atención a sus propios vecinos; además, dicho presupuesto debe servir para capacitar continuamente al personal en temas de gestión de cultura mediante convenios o por sí mismas para que su personal se adecue a las nuevas tecnologías y de su sociedad.

Las municipalidades analizadas deben mejorar sus comunicaciones entre ellas sin recelo y no deben enclaustrarse en su propio distrito 
sino deberían compartir, observar y ver cómo pueden mejorar sus servicios viendo a los distritos aledaños y crear nuevas actividades, esto da una mejora notable y abre un desarrollo en el área de cultura.

Las municipalidades analizadas deberían tener un equipo que analice y evalúe las actividades para saber y conocer a su público, y poder ofrecer una mejor calidad de servicio en su comunidad.

Finalmente, las municipalidades analizadas deben mejorar e implementar medios informativos impresos, digitales y audiovisuales para que estén más cerca de sus vecinos. Además, crear un programa de creación de público que esté enfocada a todas las edades, es especial para la niñez y adolescencia con el fin de instruirles el consumo de la cultura.

\section{REFERENCIAS BIBLIOGRÁFICAS}

Antoine, C. (2011). La sociedad del ocio y el consumo cultural. Blog de Cristian Antoine. Recuperado de http://cristian-antoine.blogspot. com/2011/08/la-sociedad-del-ocio-y-el-consumo.html

Arévalo, J. \& Cerro, S. (2004). Benchmarking: una herramienta para gestionar la excelencia en las bibliotecas y los servicios de información. Universidad de Burgos. Recuperado de http:// eprints.rclis.org/4963/1/5.htm

Deriche, Y. (2006). Selección de lecturas sobre promoción cultural. Recuperado de http://www. cnsc.cult.cu/wp-content/uploads/publicaciones/textos/Seleccion_de_lecturas_pc.pdf

Instituto Nacional de Estadística e informática (2016). Planos estratificados de Lima Metropolitana a Nivel de Manzana 2016. INEI: Lima. https://www.inei.gob.pe/media/MenuRecursivo/ publicaciones_digitales/Est/Lib1403/libro.pdf

Lima Cómo Vamos (2017). Encuesta de Lima cómo vamos 2016. Recuperado de: http:// www.limacomovamos.org/cm/wp-content/ uploads/2017/04/EncuestaLimaC\%C3\%B3moVamos_2016.pdf

Losada, C. (1999). ¿De burócratas a gerentes? Las ciencias de la gestión aplicadas a la administración del estado. Washington: Editorial Banco Interamericano de Desarrollo. Recuperado de: https://publications.iadb.org/publications/ spanish/document/\%C2\%BFDe-bur\%C3\%B3cratas-a-gerentes-Las-ciencias-de-la-ges-
ti\%C3\%B3n-aplicadas-a-la-administraci\%C3\%B3n-del-Estado.pdf

Luengo, M. (2008). El producto cultural: claves epistemológicas de su estudio. Revista Zer, 13(24), 317-335. Recuperado de: https://ojs.ehu.eus/ index.php/Zer/article/view/3630/3262

Ministerio de cultura (2012). Lineamientos de la política cultural 2013 - 2016. Recuperado de: https:// www.cultura.gob.pe/sites/default/files/pagbasica/tablaarchivos/11/lineamientomc.pdf

Ministerio de Cultura de Colombia (2013). Herramientas para la gestión cultural pública. Recuperado de: https://www.mincultura.gov.co/ areas/fomentoregional/Documents/ManualGestion_optimized_Final_11_06_13.pdf

Ministerio de Economía y Finanzas (2018). Información económica. Recuperado de: http://apps5. mineco.gob.pe/transparencia/Navegador/default.aspx

Municipalidad de Ate (2008). Cuadro de asignación de personal (CAP) (aprobado con Ordenanza $\mathrm{N}^{\circ}$ 198-MDA). Lima, Perú.

Municipalidad de Ate (2015). Plan Estratégico Institucional 2015-2018. Recuperado de: http:// www.muniate.gob.pe/ate/files/documentosPlaneamientoOrganizacion/PEI/20152018/ PEI20152018.pdf

Municipalidad de Ate (2016). Ordenanza N ${ }^{\circ} 409$ MDA aprueba la organización, número de delegados, mecanismos de elección del comité distrital del patrimonio cultural de Ate. Recuperado de: www.muniate.gob.pe/ate/verNorma.php?id=1585

Municipalidad de Ate (2018) Memoria institucional 2017 (Aprobado con Acuerdo de Consejo $N^{\circ}$ 036-2018/MDA). Lima, Perú.

Municipalidad de Miraflores (2011). Cuadro para asignación de personal (CAP). Recuperado de http://www.miraflores.gob.pe/Gestorw3b/ files/pdf/7561-19178-cap-2011---miraflores1. pdf

Municipalidad de Miraflores (2018). Informe Memoria Anual 2017. Lima, Perú.

Municipalidad de Miraflores (s/f). Plan Estratégico Institucional 2011-2015. Recuperado de: https://www.miraflores.gob.pe/wpcontent/ uploads_pdf/Plan_Estrategico_Institucional_1_hasta_65.pdf

Municipalidad de San Isidro (2015). Plan Estratégico Institucional. Periodo 2015-2017. Recuperado de: www.munisanisidro.gob.pe/transparencia/tema02/PEI_2015-2017.pdf 
Municipalidad de San Isidro (2018). Memoria Institucional 2018. Lima, Perú.

Municipalidad de San Isidro (2019). Cuadro de Asignación de personal provisional. Recuperado de: www.munisanisidro.gob.pe/Transparencia/ Tema02/Ordenanza-510-2019_CAP.pdf 
